\title{
QUALITY MANAGEMENT TOOLS IN A REFERENCE CENTER IN BREAST DIAGNOSIS: A PILOT STUDY
}

Ilse Franco de Oliveira', Camila Leal Diniz¹, Rosemar Macedo Sousa Rahal'1, Danielle Cristina Silva Roriz¹, Rosangela da Silveira Corrêa ${ }^{1}$, Ruffo de Freitas-Junior ${ }^{1}$

'Universidade Federal de Goiás - Goiânia (GO), Brazil.

Objective: To develop a model for Quality Management in a Reference Center for breast diagnosis. Methodology: Crosssectional study of an interpretative nature of qualitative research based on research and management models. Results: A survey of the priority problems and the respective primary actions required was carried out with the construction of the Zero Base Goals matrix to analyze the three sources of losses for the Image sector. The Anomaly Report model was developed to identify nonconformities, their possible causes, as well as their analysis and action plans for standardization. Then, the standard operating procedures for the assignments of the monitoring and quality assurance groups of the images were built. The standard operating procedure of quality control for risk management with its possible failures and preventive actions was also prepared, as well as the form for monitoring the acquisition and processing of images, the form for monitoring and analyzing rejected images, and their respective failures. In a later stage, the technical quality standards were prepared for the phase of execution of mammographic examinations, following the quality standards determined by the regulatory agencies. For the execution phase, which comprises the stages of experimentation and development of action proposals for improvement or adaptation, some changes will be made to improve the process. Conclusion: The development of the Quality Management model in a Reference Center for breast diagnosis is in the phase of correction and adaptation for subsequent application, allowing for timely and relevant changes as the corresponding needs are evidenced.

Keywords: Breast; Diagnosis; Quality Management. 